\author{
R. DIETSCHE ${ }^{1}$ \\ D.C. LIM ${ }^{2}$ \\ M. BUBEK ${ }^{1}$ \\ I. LOPEZ-SALIDO \\ G. GANTEFÖR ${ }^{1}$ \\ Y.D. $\mathrm{KIM}^{3, \text {, }}$
}

\title{
Comparison of electronic structures of mass-selected Ag clusters and thermally grown Ag islands on sputter-damaged graphite surfaces
}

\footnotetext{
${ }^{1}$ Department of Physics, University of Konstanz, 78457 Konstanz, Germany

${ }^{2}$ Department of Surface Technology, Korea Institute of Materials Science, Changwon, Korea

${ }^{3}$ Department of Chemistry, Sungkyunkwan University, 440-746 Suwon, Korea
}

\begin{abstract}
Ag cluster anions consisting of 3-16atoms were deposited on sputter-damaged HOPG surfaces using a soft-landing technique (mean deposition energy less than $0.2 \mathrm{eV} /$ atom) at room temperature. For investigations of the structures of deposited clusters, X-ray photoelectron spectroscopy (XPS) and scanning tunneling microscopy (STM) were used. In addition, the chemical properties of deposited clusters were studied using atomic oxygen and $\mathrm{CO}$. Comparison of the properties of deposited $\mathrm{Ag}$ clusters and $\mathrm{Ag}$ islands with similar sizes grown by evaporating $\mathrm{Ag}$ atoms on the same substrate shows different results, implying that two different preparation methods give either different shapes of $\mathrm{Ag}$ clusters and islands, or dissimilar metal-support interactions.
\end{abstract}

PACS 73.22.-f

\section{$1 \quad$ Introduction}

Recent developments into the deposition of massselected clusters has opened new insights into the sizeselectivity of physical and chemical properties of clusters. In contrast to the nanoparticles prepared by thermal evaporation of metal atoms on a substrate, deposition of size-selected clusters can yield highly mono-disperse surface structures, which cannot be obtained using other techniques. Therefore, precise information about the size-selectivity of electronic and chemical properties can be gained using size-selected clusters. Pioneering works by Heiz's group found strong size dependence of the catalytic activity of mass-selected $\mathrm{Au}, \mathrm{Ni}, \mathrm{Pd}$ and Pt clusters on various substrates [1-5]. Structural properties and catalytic activity of $\mathrm{Au}_{n} / \mathrm{TiO}_{2}(n=$ number of atoms in a cluster) systems were investigated by Anderson's and Burrato's groups [6,7]. In these previous works, clusters consisting of less than 20-30 atoms were deposited on various oxide surfaces with relatively low kinetic energies (less than $\sim 1-2 \mathrm{eV}$ /atom).

Highly-ordered pyrolytic graphite (HOPG) was shown to be a useful substrate for preparing metallic nanostructures, since surface preparation (cleaning) is relatively easy due to its inert surface properties [8-11]. Studies on electronic

\Fax: +82-31-290-7075, E-mail: ydkim91@skku.edu and chemical properties of metallic nanostructures formed on carbon substrates have implications in electrochemistry and heterogeneous catalysis, and HOPG serves as a useful substrate for model systems. Preparation of highly monodisperse surface structures on HOPG is difficult when low kinetic energies are used for cluster deposition: the sticking probabilities of metal atoms and clusters on nearly perfect HOPG surfaces are too low, and the soft-landed metal clusters on HOPG are too mobile at room temperature, yielding agglomeration of the clusters. If cluster agglomeration takes place, the size-dependence of the cluster properties cannot be studied. To avoid this problem, Palmer and co-workers deposited $\mathrm{Ag}, \mathrm{Au}, \mathrm{Ni}$ and $\mathrm{Pd}$ clusters with higher impact energies ( $\gg 10 \mathrm{eV} /$ atom) on HOPG surfaces [12-16]. Metal clusters with high kinetic energies create defects on HOPG surfaces and are subsequently pinned on those defects. In this work, we soft-landed Ag clusters (mean kinetic energy of deposited clusters of less than $0.2 \mathrm{eV} /$ atom) on mildly sputtered HOPG surfaces, on which diffusion of Ag clusters at room temperature is inhibited, most likely due to the stronger interactions between $\mathrm{Ag}$ and the substrate, compared to those on non-defect surfaces. Electronic and chemical properties of deposited $\mathrm{Ag}$ clusters smaller than $\mathrm{Ag}_{17}$ were studied using $\mathrm{X}$-ray photoelectron spectroscopy (XPS). It is suggested that structures of soft-landed clusters are different from those of $\mathrm{Ag}$ "islands", which are grown by thermal evaporation of $\mathrm{Ag}$ atoms on the surface. This result implies that dissimilar structures of differently prepared surfaces should be taken into account, when properties of deposited clusters and thermally grown islands are compared.

2

\section{Experimental}

Details of the experimental set-up and oxidation/ reduction experiments can be found elsewhere [17]. HOPG samples were outgassed at $800 \mathrm{~K}$ for at least $12 \mathrm{~h}$. Subsequently, the HOPG surfaces were sputtered by Ar ions with a kinetic energy of $0.5 \mathrm{keV}$ for $20 \mathrm{~s}$, in order to create defect sites for stabilizing Ag clusters on the surface (sample current during sputtering $=1-2 \mu \mathrm{A}$ ). The clusters are produced by a magnetron sputter source [18]. After extracting the ion beam into vacuum, the cluster anions were accelerated to $1.5 \mathrm{kV}$. Mass separation was done by a $30^{\circ}$ sector magnet with a resolution of about $m / \Delta m=20$. In front of the sample, clusters 
were decelerated to the desired deposition energy by applying the corresponding voltage to the sample. The number of clusters deposited on the surface can be counted by measuring the sample current during deposition.

For oxidation of $\mathrm{Ag}$ clusters on HOPG, the chamber was backfilled by $\mathrm{O}_{2}$ with a pressure of $8 \times 10^{-5} \mathrm{mbar}$, and at the same time, a hot Pt-filament was placed on the backside of the sample (referred to as "atomic oxygen"). The exposure time of each sample to atomic oxygen was $10 \mathrm{~min}$. This method is known to create atomic oxygen environments, even though the existence of excited molecular oxygen species cannot be completely excluded.

For scanning tunneling microscopy (STM) studies, samples were transferred from the XPS/deposition-chamber to a separate room temperature STM (OMICRON 1) chamber using a portable ultrahigh vacuum (UHV)-system. For STM imaging, a constant current mode was used with Pt/Ir tips.

\section{3}

\section{Results and discussion}

Figure 1 shows the $\mathrm{Ag} 3 d$ XPS spectra of $\mathrm{Ag}_{13}$ clusters soft-landed on sputtered HOPG surfaces (mean deposition energy: $2.6 \mathrm{eV}$ ). At the initial stage of $\mathrm{Ag}$ deposition, the intensity of the $\mathrm{Ag} 3 d$ peak grows with increasing number of deposited clusters without much alteration of the widths and positions of the peaks. Only when the number of clusters deposited increases from $4 \times 10^{12}$ to $8 \times 10^{12}$ (the highest $\mathrm{Ag}$ coverage in Fig. 1), the $\mathrm{Ag} 3 d$ state becomes narrower by $0.2 \mathrm{eV}$, and the peak center is shifted to a lower binding energy. One possible interpretation of the data is that the $\mathrm{Ag}$ clusters exist as individual entities at lower Ag coverages, whereas at larger coverages $\left(>4 \times 10^{12}\right)$, agglomeration starts. Our STM result suggests an Ag coverage below $10 \%$, even though the coverage determination is crude due to the inhomogeneity of the cluster spot. We conclude that as long as the number of deposited $\mathrm{Ag}_{13}$ is below $4 \times 10^{12}, \mathrm{Ag}$ clusters can be assumed to exist as individual clusters on the surface. Note that the core level spectrum is quite sensitive to the particle size: with increasing particle size, the full-width-half-maximum (FWHM) decreases and the peak shifts to the lower binding energy [8, 19]. If no agglomeration would take place at high coverages, the $\mathrm{Ag} 3 d$ peak width should remain constant, or increase with increasing Ag coverage due to the occupation of

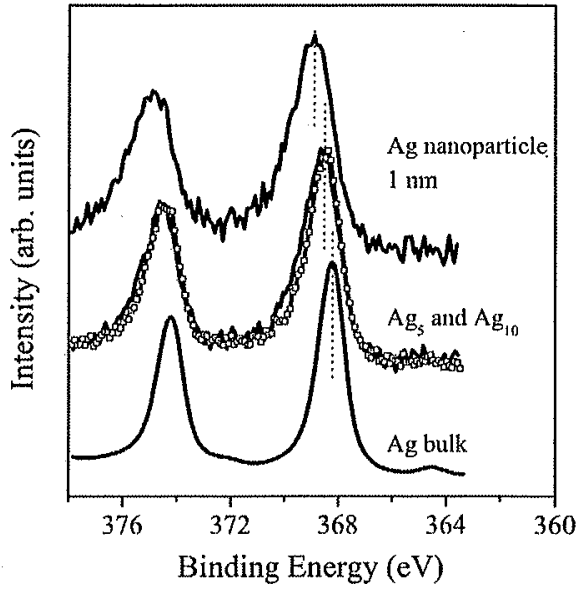

FIGURE $2 \mathrm{Ag} 3 d$ level spectra of $\mathrm{Ag}$ bulk, deposited $\mathrm{Ag}_{5}$ and $\mathrm{Ag}_{10}$ (spectrum with squares) clusters on sputter-damaged HOPG, and $1 \mathrm{~nm}$-sized Ag islands prepared using thermal evaporation of $\mathrm{Ag}$ atoms on the same substrate are compared

various adsorption sites by $\mathrm{Ag}$ clusters at higher coverages. Decrease of the FWHM of the Ag $3 d$ peaks upon agglomeration can be rationalized by the lower surface to volume ratio of a larger particle, since a smaller relative surface area will lead to the lower heterogeneity of the chemical environment of $\mathrm{Ag}$ atoms in a cluster. In addition, the broadening of the $\mathrm{Ag} 3 d$ core levels for smaller metal particles has been also found in a previous study and attributed to the different life time broadenings of the hole state for different sized particles [20].

In order to study variation of the electronic structure of $\mathrm{Ag}$ as a function of cluster size, $\mathrm{Ag}_{n}$ with $n=3-16$ were soft-landed on sputter-damaged HOPG surfaces, and an Ag $3 d$ core level spectrum of each sample was taken (number of clusters deposited for each sample $=4 \times 10^{12}$ ). In Fig. 2, $\mathrm{Ag} 3 d$ spectra of $\mathrm{Ag}_{5}$ and $\mathrm{Ag}_{10}$ are shown. Depending on the cluster size, different binding energies of the Ag $3 d$ level are expected, yet no significant difference in the $\mathrm{Ag} 3 d$ binding energy can be found among different clusters. Except for $\mathrm{Ag}_{4}$ and $\mathrm{Ag}_{5}$, all $\mathrm{Ag}$ clusters show the same $\mathrm{Ag} 3 d$ binding energies. $\mathrm{Ag}_{4}$ and $\mathrm{Ag}_{5}$, showing almost identical $\mathrm{Ag} 3 d$ peaks, differ from other $\mathrm{Ag}$ clusters in terms of the $\mathrm{Ag} 3 d$ binding energy and the peak width: however, the difference is compa-

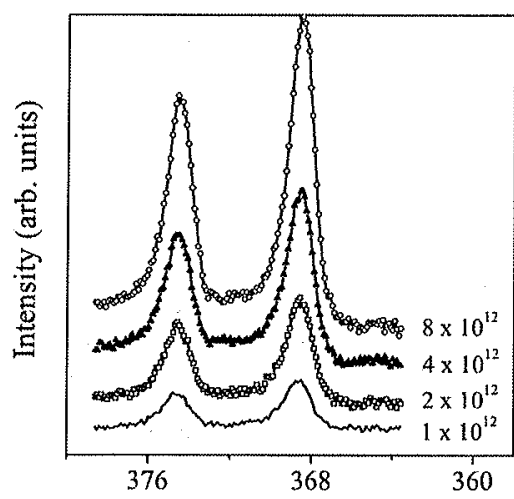

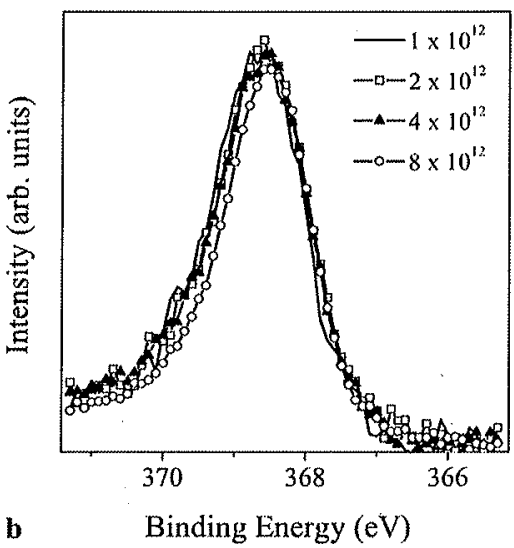

FIGURE 1 (a) $\mathrm{Ag} 3 d$ core level spectra of $\mathrm{Ag}_{13}$ clusters deposited on sputter-damaged HOPG surfaces. The number of clusters estimated by measuring the sample current for each sample is given in the figure. (b) shows a magnified view of the same spectra shown in (a), in which each spectrum was normalized 


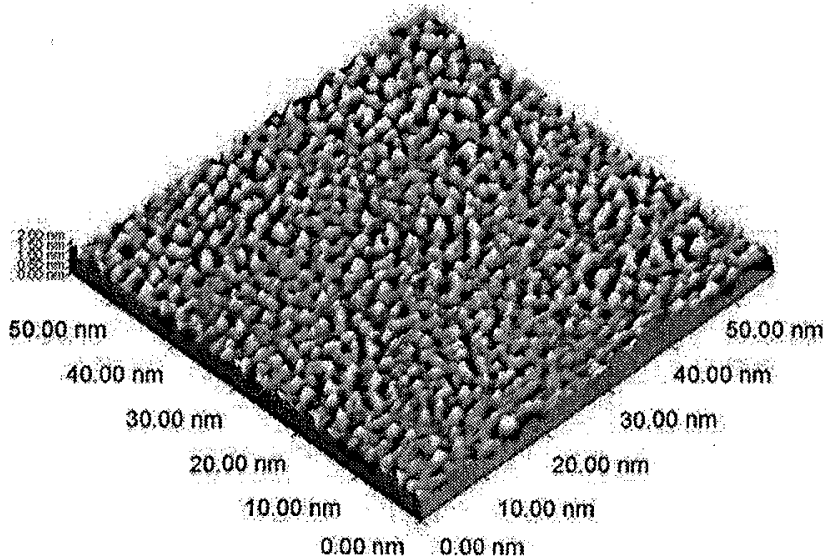

FIGURE 3 Constant current STM image of $\mathrm{Ag}_{13}$ clusters $\left(4 \times 10^{12}\right)$ deposited on the sputter-damaged HOPG. Tunneling parameters are $0.4 \mathrm{~V}$ and $0.8 \mathrm{nA}$

rable to the upper limit of our instrumental resolution, which is estimated to be $0.1 \mathrm{eV}$ (Fig. 2).

As previously shown, the $\mathrm{Ag} 3 d$ binding energies are quite sensitive to the Ag particle size on graphite. We have previously found, using STM in combination with XPS, that the $\mathrm{Ag} 3 d$ level almost linearly increases with decreasing particle diameter, i.e. Ag particle size can be estimated by measuring $\mathrm{Ag} 3 d$ binding energy. For the particles with a diameter of $1 \mathrm{~nm}$ (less than $\sim 30$ atoms in a particle), the core level shift with respect to the bulk value amounts to about $0.6 \mathrm{eV}$ (Fig. 2) $[8,10]$. Since the $\mathrm{Ag} 3 d$ binding energies of the deposited $\mathrm{Ag}$ clusters are rather similar to the $\mathrm{Ag}$ islands of about $5 \mathrm{~nm}$ in diameter, one may argue that Ag clusters agglomerate forming larger particles. However, we can exclude the possibility of sintering based on the following results:

1) Our STM image of $\mathrm{Ag}_{13}$ clusters suggests that the clusters remain as individual entities after deposition as long as the number of $\mathrm{Ag}$ clusters deposited is smaller than $4 \times 10^{12}$ (Fig. 3). No significant agglomeration can be found in our STM image. We have recently shown that Au clusters soft-landed on the same substrate mostly remain as individual entities [17]. In that study, in addition to the STM study, oxidation behaviors of mass-selected deposited clusters were studied, which turned out to be strongly size-selective [17]. The height of each cluster is less than $0.5 \mathrm{~nm}$, and the width is estimated to be smaller than $1 \mathrm{~nm}$ (Fig. 3). It is worth mentioning that the width of the cluster cannot be precisely determined due to the tip-cluster convolution [21].

2) We oxidized the $\mathrm{Ag}$ clusters using atomic oxygen and made attempts to reduce them with $\mathrm{CO}$. In the case of $\mathrm{Ag}$ nanoparticles with a diameter of $5 \mathrm{~nm}$, the oxidized $\mathrm{Ag}$ nanoparticles can be partially reduced by $\mathrm{CO}$ under the same experimental conditions. However, in the case of massselected deposited clusters, such a reduction by $\mathrm{CO}$ cannot be found (Fig. 4). This result is rather similar to the oxidation/reduction behaviors of $\mathrm{Ag}$ nanoparticles smaller than 3-4 nm [10, 22]. Recently, the inertness of the small oxidized $\mathrm{Ag}$ nanoparticles on HOPG has been attributed to $\mathrm{Ag}$ carbonate formation [22]. Taking both electronic and chemical properties of deposited Ag clusters into account, deposited mass-selected clusters are dissimilar to Ag nanoparticles grown by evaporating $\mathrm{Ag}$ atoms on the sputtered HOPG with any sizes.

Deposition of Ag clusters on HOPG using higher kinetic energy than ours found no indication of fragmentation [12-16]. Considering the low mean deposition energy of $0.2 \mathrm{eV}$ per atom we used for soft-landing, cluster fragmentation is not likely.

For $\mathrm{Ag}$ islands prepared by evaporating $\mathrm{Ag}$ atoms on the sputtered HOPG surface, it was previously shown that the $\mathrm{Ag} 3 d$ binding energy is very sensitive to the particle size: with decreasing particle size, a positive core level shift can be found, resulting in a shift of $0.6 \mathrm{eV}$ for about $1 \mathrm{~nm}$-sized particles [10]. For understanding the size dependent positive core level shift, both initial and final state effects should be taken into account. Regarding the initial state effect, we previously found that there is a partial charge transfer from $\mathrm{Ag}$ to carbon, leading to a positive core level shift [10]. In addition, other factors such as the change of the lattice constant with decreasing particle size, and metal-insulator-transition, can come into play $[8,10]$. When particle size becomes smaller, the positive hole created by the photoionization process can be less efficiently screened, resulting in an additional positive core level shift (final state effect). The large difference in the core level shifts of the deposited mass-selected clusters (core level shift of $0.2 \mathrm{eV}$ ) and the similarly sized Ag islands (core level shift of $0.6 \mathrm{eV}$ ) indicates that $\mathrm{Ag}$ atoms in both species are in different chemical environments. For rationalizing this result, one may suggest different shapes of similarly sized
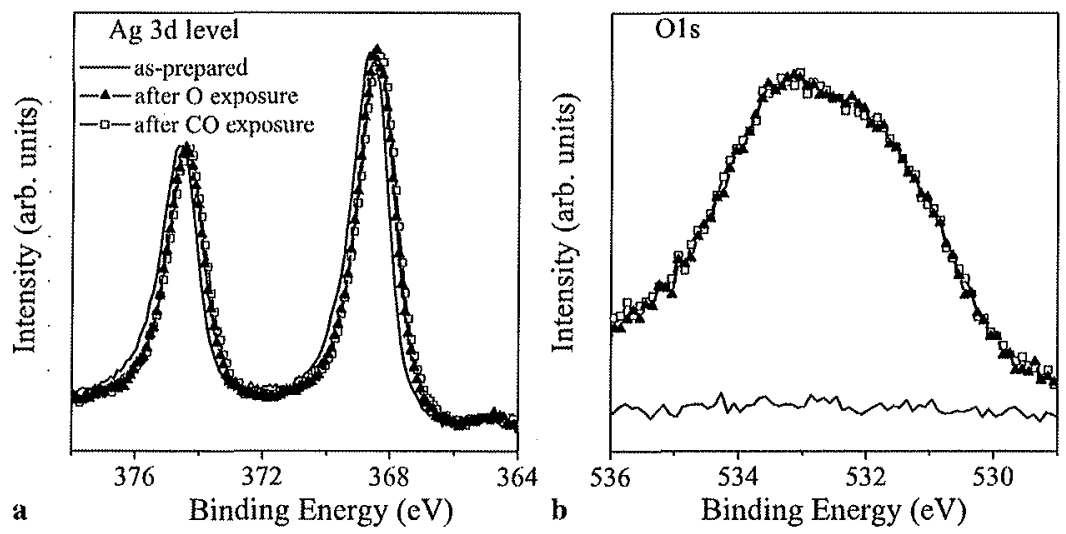

FIGURE 4 (a) $\mathrm{Ag} 3 d$ and (b) $\mathrm{O} 1 s$ core level spectra of soft-landed $\mathrm{Ag}_{13}$ clusters $\left(4 \times 10^{12}\right)$ after deposition, after exposing to atomic oxygen, and after subsequent exposure to $\mathrm{CO}\left(3000 \mathrm{~L}, 1 \mathrm{~L}=1 \times 10^{-6} \mathrm{Torrs}\right)$ are demonstrated 
clusters and islands. Depending on the preparation method of Ag clusters (or islands) with a specific size, different isomers can form, resulting in various core level shifts. Formation of different isomers is possible, if the cluster or island formation is limited by kinetics rather than dominated by thermodynamic stability [23]. One can imagine that the metal-metal bond length is different in various isomers, and this can result in diverse rehybridization of the valence band structure, leading to different core level shifts [24].

Another scenario, which cannot be ruled out, is that new defects are created on the sputtered HOPG surfaces after softlanding, on which clusters are pinned or implanted. The electronic structure of $\mathrm{C}$ should change depending on the local structure, e.g. comparing to the $s p^{2}$-network, extended defects with $s p^{3}$-like structure should show much lower work functions, which has a propensity toward being electron-donor rather than an electron-acceptor [25]. Therefore, different defect structures can cause different metal-support interactions. Further studies to prove different metal-support interactions are essential.

Previously, we found a strong size dependence of the chemical properties of mass-selected Au clusters deposited on sputter-damaged HOPG [17]. In contrast, in the present work, no significant change of the oxidation behaviors of mass-selected $\mathrm{Ag}$ clusters deposited on the same substrate was found. The atomic oxygen exposure of $\mathrm{Ag} / \mathrm{HOPG}$ forms Ag-carbonate, i.e. the strong metal-support interaction is important, and the size of $\mathrm{Ag}$ clusters deposited on HOPG is not crucial for determining oxidation behaviors of $\mathrm{Ag}$ on sputtered HOPG [22].

\section{4}

\section{Summary}

Mass-selected Ag clusters were deposited on sputter-damaged HOPG surface. Our results suggest that clusters stay as individual entities with minor agglomeration upon deposition. Different electronic properties of deposited $\mathrm{Ag}$ clusters, comparing to similarly sized $\mathrm{Ag}$ islands grown by evaporating $\mathrm{Ag}$ atoms onto the surface, were found in the $\mathrm{Ag}$ $3 d$ core level spectra. No size-dependence of the chemical properties of Ag clusters could be observed.

ACKNOWLEDGEMENTS We acknowledge the German Research Foundation (DFG) for financial support within SFB (Sonder- forschungsprogram) 513 project number A 15 . This work was supported by the Korean Science and Engineering Foundation (KOSEF) and grant funded by the Korea government (MOST) (R01-2007-000-10280-0).

\section{REFERENCES}

1 B. Yoon, H. Häkkinen, U. Landman, A.S. Wörz, J.M. Antonietti, S. Abbet, K. Judai, U. Heiz, Science 307, 403 (2005)

2 K. Judai, S. Abbet, A.S. Wörz, U. Heiz, C.R. Henry, J. Am. Chem. Soc. 126, 2732 (2004)

3 A.S. Wörz, K. Judai, S. Abbet, U. Heiz, J. Am. Chem. Soc. 125, 7964 (2003)

4 U. Heiz, A. Sanchez, S. Abbet, W.-D. Schneider, J. Am. Chem. Soc. 121, 3214 (1999)

5 U. Heiz, F. Vanolli, A. Sanchez, W.-D. Schneider, J. Am. Chem. Soc. 120, 9668 (1998)

6 S. Lee, C.Y. Fan, T.P. Wu, S.L. Anderson, J. Chem. Phys. 123, 124710 (2005)

7 X. Tong, L. Benz, P. Kemper, H. Metiu, M.T. Bowers, S.K. Buratto, J. Am. Chem. Soc. 127, 13516 (2007)

8 I. Lopez-Salido, D.C. Lim, Y.D. Kim, Surf. Sci. 588, 6(2005)

9 D.C. Lim, I. Lopez-Salido, Y.D. Kim, Surf. Sci. 598, 96 (2005)

10 I. Lopez-Salido, D.C. Lim, R. Dietsche, N. Bertram, Y.D. Kim, J. Phys. Chem. B 110, 1128 (2006)

11 D.C. Lim, I. Lopez-Salido, R. Dietsche, M. Bubek, Y.D. Kim, Surf. Sci. $600,507(2006)$

12 S.G. Hall, M.B. Nielsen, R.E. Palmer, J. Appl. Phys. 83, 733 (1998)

13 S.J. Carroll, S.G. Hall, R.E. Palmer, R. Smith, Phys. Rev. Lett. 81, 3715 (1998)

14 S.J. Carroll, S. Pratontep, M. Streun, R.E. Palmer, S. Hobday, R. Smith, J. Chem. Phys. 113, 7723 (2000)

15 S. Gibilisco, M. Di Vece, S. Palomba, G. Faraci, R.E. Palmer, J. Chem. Phys. 125, 084704 (2006)

16 M. Di Vece, S. Palomba, R.E. Palmer, Phys. Rev, B 72, 073407 (2005)

17 D.C. Lim, R. Dietsche, M. Bubek, T. Ketterer, G. Ganteför, Y.D. Kim, Chem. Phys. Lett. 439, 364 (2007)

18 H. Haberland, M. Mall, M. Moseler, Y. Qiang, T. Reiners, Y. Thurner, J. Vac. Sci. Technol. A 12, 2925 (1994)

19 V.I. Bukhtiyarov, A.F. Carley, L.A. Dollard, M.W. Roberts, Surf. Sci. 381, L605 (1997)

20 A. Sandell, J. Libuda, P.A. Brühwiler, S. Andersson, A.J. Maxwell, M. Bäumer, N. Mårtensson, H.J. Freund, J. Vac. Sci. Technol, A 14, 1546 (1996)

21 H. Hövel, T. Becker, A. Bettac, B. Reihl, M. Tschudy, E.J. Williams, J. Appl. Phys. 81, 154 (1997)

22 S.H. Jeong, D.C. Lim, J.-H. Boo, S.B. Lee, H.N. Hwang, C.C. Hwang Y.D. Kim, Appl. Catal. A 320, 152 (2007)

23 H. Häkkinen, M. Moseler, U. Landman, Phys. Rev. Lett. 89, 033401 (2002)

24 B. Richter, H. Kuhlenbeck, H.-J. Freund, P.S. Bagus, Phys. Rev. Lett. 93 , 026805 (2004)

25 A.N. Obraztsov, A.P. Volkov, A.I. Boronin, S.V. Kosheev, Diam. Relat. Mater. 11, 813 (2002) 